\title{
Implementation of Project Evaluation and Review Technique (PERT) and Critical Path Method (CPM): A Comparative Study
}

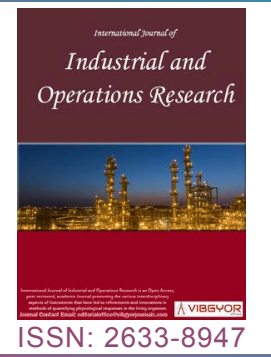

\section{Orumie Ukamaka Cynthia*}

Department of Mathematics/Statistics, University of Port Harcourt, Nigeria

\begin{abstract}
The project evaluation and review technique (PERT) and critical path method (CPM) were applied in a building construction company. Different activities involved in the house construction project were described. The earliest events, latest commencement and completion of activities were determined using Forward and Backward pass computations in CPM. Critical paths were determined using both CPM and PERT. Based on the analysis, it was shown that the completion of the house project using CPM was almost the same with that of PERT such that the difference between both techniques was only one day. The probability that the house construction project could be completed in 151 days was $99.87 \%$. This implies that the house construction project could be completed on time using both techniques. However, the results showed that the implementation of both CPM and PERT methods are effective and efficient in a house construction project.
\end{abstract}

Keywords

Project evaluation and review technique, Critical path method, Earliest events, Activities, project

\section{Introduction}

Project Evaluation and Review Technique (PERT) and Critical Path Method (CPM) are scheduling methods originally designed to plan a construction project and to analyse and represent the tasks involved in completing a given project by employing a network of associated and similar activities, coordinating optimum cost and time criteria [1]. According to Kelly and Walker [2], Critical Path Method (CPM) and Programme Evaluation and Review Technique (PERT) are useful tools for efficient management of all types of project such as construction, engineering, facility maintenance, research and development.

However, the problem of delays in the construction industry is a global phenomenon. Project management in construction project with CPM/PERT is one of the most challenging jobs that any manager can take on which involves, the management of a large-scale project that requires coordinating numerous activities throughout the organization $\mathrm{Li}$, et al. [3].

In the field of construction, scheduling is important because it sets time and sequence of the various stages, the linkage between one activity to another to ensure that the deadline can be achieved.

*Corresponding author: Orumie Ukamaka Cynthia, Department of Mathematics/Statistics, University of Port Harcourt, Nigeria, Tel: 08064099683

Accepted: February 29, 2020; Published: March 02, 2020

Copyright: (C 2020 Cynthia OU. This is an open-access article distributed under the terms of the Creative Commons Attribution License, which permits unrestricted use, distribution, and reproduction in any medium, provided the original author and source are credited.

Cynthia et al. Int J Ind Operations Res 2020, 3:004 
CPM and PERT allow one to identify this important task and stay on track throughout the entire construction project [4]. CPM technique is used based on the knowledge and experience of the past projects for predicting accurately the time required for various activities during the execution of the project. The company under consideration uses CPM and PERT, and hierarchy of their already completed construction project events are well defined and time of completion.

Therefore, here, CPM and PERT are applied to a house construction project and the earliest events, the latest commencement and completion of activities of a house construction project is calculated. The critical path in the house construction project is also to be determined as well as evaluating the differences existing between CPM and PERT.

Section two is the brief history of the construction company used. Section three presents brief explanations of CPM, PERT and their difference. Data collection/methodology and analyses are described in Section four and five respectively. Section six gives summary and conclusion.

\section{Brief History of the Company}

Basse Engineering Construction Company is a dynamic construction company with more than twenty five years of experience in pursuing excellence in the delivery of Civil Engineering and construction services.

The Company specializes in public and private house building, maintenance, design and construction of highways and bridges, town planning and construction of modern housing units, erosion control works, underground drainage system, construction of sewage system, etc.

According to the manager, the manages scheduled construction project delivery on time to avoid delays and cost overrun. It has set construction standards in both Nigeria and outside country.

Basse Engineering Construction Company is located at No. 713 Idoro Road, Uyo Akwa Ibom State. Its distance is about $3.5 \mathrm{~km}$ away from Itam Junction from the East and adjacent to the new stadium Road, off Abak Road, Uyo. Their project site was established in 1994 with skilled professionals and highly experienced Engineers.
(CPM) Critical Path Method, PERT (Program Evaluation and Review Technique) and Their Differences

\section{(CPM) Critical Path Method}

CPM provides planned schedule to assist the project team and forms the basis for checking project schedule performance by comparing actual with planned task progress. CPM is a means of evaluating how long will each task take before one can finish the entire project Hebert, et al. [5].

It plans and controls a large number of activities that have complex dependencies on design and construction issues requiring time and cost functions. The time estimate used in CPM denotes the normal time, and links to the trade-off between completion time and the costs of the project [6].

However, CPM can be defined as a sequence of project network activities that add up to the longest duration. It's sequence regulates the least time possible to complete the project.

Critical Path Method assist in the management of projects in two different ways: The forward and backward pass.

The forward pass calculation is obtained with the formula below:

$$
E S j=\operatorname{Maxj}\{E S i+D i j\} \quad v i j
$$

The forward calculation moves from the initial event to the terminal event, setting the initial event at zero. i.e., $E i=0$

Where;

$E T$ : Earliest event or activity time

$E i$ or ESi : The earliest start of activity

$E j$ or $E S j:$ The latest start event

Implying, $E S j=L C j-D i j$

$D i j$ : The time required for an activity.

After the forward pass computation, the earliest completion and the latest completion of activities are obtained from the backward pass computation with the formula stated below:

$$
L C j=\operatorname{Min} i\{L C j-D i j\} \quad V i j \text { activities }
$$

Where;

$\mathrm{Li}$ or $\mathrm{LCi}$ : The earliest completion of an activity 
$L j$ or $L C j$ : The latest completion of an activity

$L C i+D i j$

\section{PERT (Program Evaluation and Review Tech- nique)}

PERT is also known as Back Research Technique. This technique uses time as a variable in planning, scheduling, organising, coordinating and controlling of uncertain activities along with performance specification.

PERT computation supports assigning probabilities with task completion times in accordance with; Optimistic time $(\alpha)$, Most likely time $(M)$ and, Pessimistic time $(\beta)$.

Determination of the critical path from PERT requires slack calculation with activities e values known as floats.

The most common floats are the total floats (TF) and free floats (FF). The critical path is determined by the values of floats $=0$. i.e., $T F=0$ and $F F=0$.

Total Floats and Free Floats are obtained from the equation below:

$$
\begin{aligned}
& T F i j=L C j-E S i-D i j \\
& F F i j=E S j-E S i-D i j
\end{aligned}
$$

That is, the slack values are also known as floats.

The mean time denoted by $(\mu T)$ and variance $(V)$ has the formula stated below:

$$
\begin{aligned}
& \text { Mean, } \mu T=\frac{(\alpha+4 M+\beta)}{6} \\
& \text { Variance, } V=\left(\frac{\beta-\alpha}{6}\right)^{2}
\end{aligned}
$$

The above follows beta distribution with parameters $((\alpha, \beta))$.

Using PERT in time predictions, the probability of completing construction time is calculated using standard normal form of beta as shown below:

$$
Z=\frac{(X-\Sigma \mu T)}{\sqrt{V_{\text {critical }}}}
$$

Where;

$\mathrm{X}=$ Total project completion time using CPM

$\Sigma \mu T_{\text {critical }}=$ Total sum of all the expected critical activities

$V_{\text {critical }}=$ Total sum of all the variances of critical activities
$\mathrm{Z}=$ Compared with tabulated normal distribution.

\section{Differences between PERT and CPM in construc- tion projects}

PERT and CMP are the two-network based project management methods that shows and exhibit the flow and sequence of activities and events. The difference between the CPM and PERT is that PERT is mainly used where the time required for completion of each of the activities is unknown whereas CPM technique bases its execution of the project on the knowledge and experience of past projects [7].

PERT technique is of planning and controlling of time and CPM is aligned towards activities.

PERT uses probabilistic model, while CPM uses a deterministic model.

There are three time estimates in PERT where as there is only one time estimate in CPM.

\section{Data Collection and Methodology}

The data was collected from the Management unit of Basse Engineering Construction Company alongside with the planning steps involved in this house construction project.

There are 15 activities and stages for the house construction project, starting from the preparatory work/site work, to the fencing work. Each activity defines the earliest start time and earliest finish time. The duration of each activity is assigned based on the secondary data stated in Table 1, gotten from the office of the manager.

A lot of records and files for already carried out projects were shown in pictures and discs as evidence to support their experience and skills.

Data for this study are analyzed using CPM and PERT methods as explained in section 3 above. They are collected as shown in Table 1 below, where each activity is labeled with codes from $A$ - $O$ and from 1 - 15 with activities description.

A project activity, description, duration, optimistic and pessimistic estimates $(a, b)$ and predecessors.

Source: file in Management unit of Basse Engineering Construction Company.

The two methods of data analysis were described in section 3 above 
Table 1: The activities and stages for the house construction project, starting from the preparatory work/site work, to the fencing work, including duration, optimistic and pessimistic estimates $(a, b)$ and predecessors.

\begin{tabular}{|l|l|l|l|l|l|l|}
\hline S/N & Activity & $\mathbf{i}-\mathbf{j}$ & Description & Duration (in days) & $\mathbf{a}, \mathbf{b}$ (in days) & Predecessor \\
\hline 1 & A & 2-Jan & Site work & 5 & 3,7 & - \\
\hline 2 & B & 3-Feb & Excavation work & 7 & 4,10 & A \\
\hline 3 & C & 4-Mar & Foundation work & 12 & 10,14 & B \\
\hline 4 & D & 6-Apr & Column structure work & 14 & 13,15 & C \\
\hline 5 & E & 5-Apr & Sloof works & 8 & 5,11 & C \\
\hline 6 & F & 8-May & Wall \& Frame works & 14 & 12,16 & E \\
\hline 7 & G & 7-Jun & Roof trust works & 12 & 8,16 & D \\
\hline 8 & H & 9-Jul & Roof (Ceiling \& Tiles) works & 19 & 12,20 & G \\
\hline 9 & I & 10-Sep & Sanitary works and Electrical & 22 & 14,24 & F, H \\
\hline 10 & J & 11-Sep & Plastering works & 27 & 24,30 & F, H \\
\hline 11 & K & 11-Oct & Floor works & 20 & 16,30 & I \\
\hline 12 & L & 12-Aug & Installation works (lamps, glass, & 17 & 16,24 & F \\
\hline 13 & M & 13-Nov & Painting works & & & \\
\hline 14 & N & 13-Dec & Fencing works & 12 & 30,24 & J, K \\
\hline 15 & O & 13-14 & Complementary works & 8 & 10,14 & L \\
\hline & & & & 6,10 & M, N \\
\hline
\end{tabular}

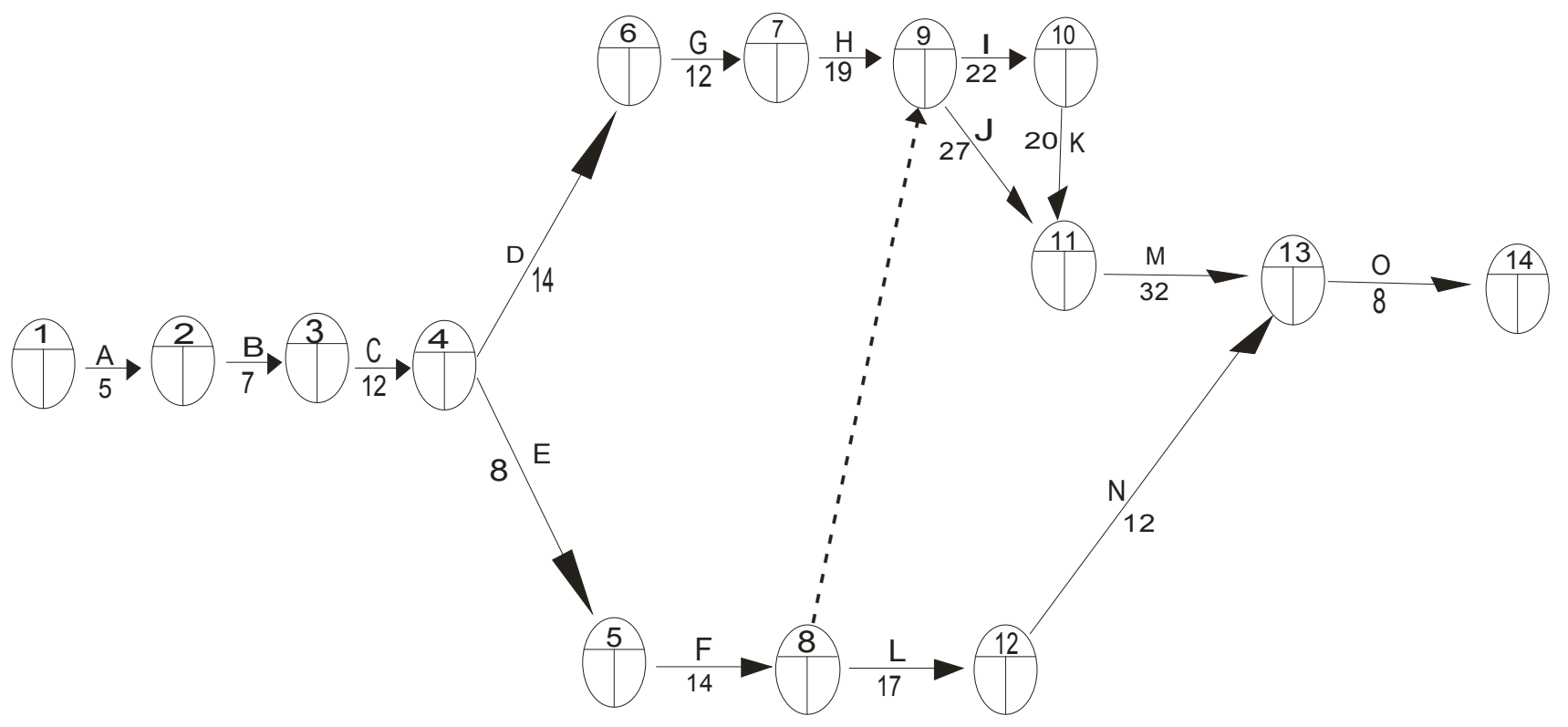

Figure 1: Network diagram with most likely estimates $(\mathrm{m})$ and durations.

Therefore the network diagram representing the data presented in Table 1 above with only most likely estimates $(\mathrm{m})$ and their corresponding durations is thus (Figure 1):

Data Analyses of a House Construction Project

\section{Data analysis with CPM and its interpretations}

The forward pass is calculated using equation (1) and is summarized in Table 2 below:

Table 2 above is the Forward Pass where the initial event, $\mathrm{E}_{1}=0$ and the terminal event, $\mathrm{E}_{14}=$ 151 as shown in row 2-column 4 and row 14-column 4 respectively. The completion of the house 
Table 2: Forward pass computation.

\begin{tabular}{|l|l|l|l|l|}
\hline Events & Immediate Preceeding Event & Duration & Earliest Activity Time & Max. \\
\hline 1 & - & - & 0 & 0 \\
\hline 2 & 1 & 5 & $0+5$ & 5 \\
\hline 3 & 2 & 7 & $5+7$ & 12 \\
\hline 4 & 3 & 12 & $12+12$ & 24 \\
\hline 5 & 4 & 8 & $24+8$ & 32 \\
\hline 6 & 4 & 14 & $24+14$ & 38 \\
\hline 7 & 6 & 12 & $38+12$ & 50 \\
\hline 8 & 5 & 14 & $32+14$ & 46 \\
\hline 9 & 7,8 & 19 & $50+19,46+0$ & 69 \\
\hline 10 & 9 & 22 & $69+22$ & 91 \\
\hline 11 & 9,10 & 20 & $69+27,91+20$ & 111 \\
\hline 12 & 8 & 17 & $46+17$ & 63 \\
\hline 13 & 11,12 & 32 & $111+32,63+12$ & 143 \\
\hline 14 & 13 & 8 & $143+8$ & 151 \\
\hline
\end{tabular}

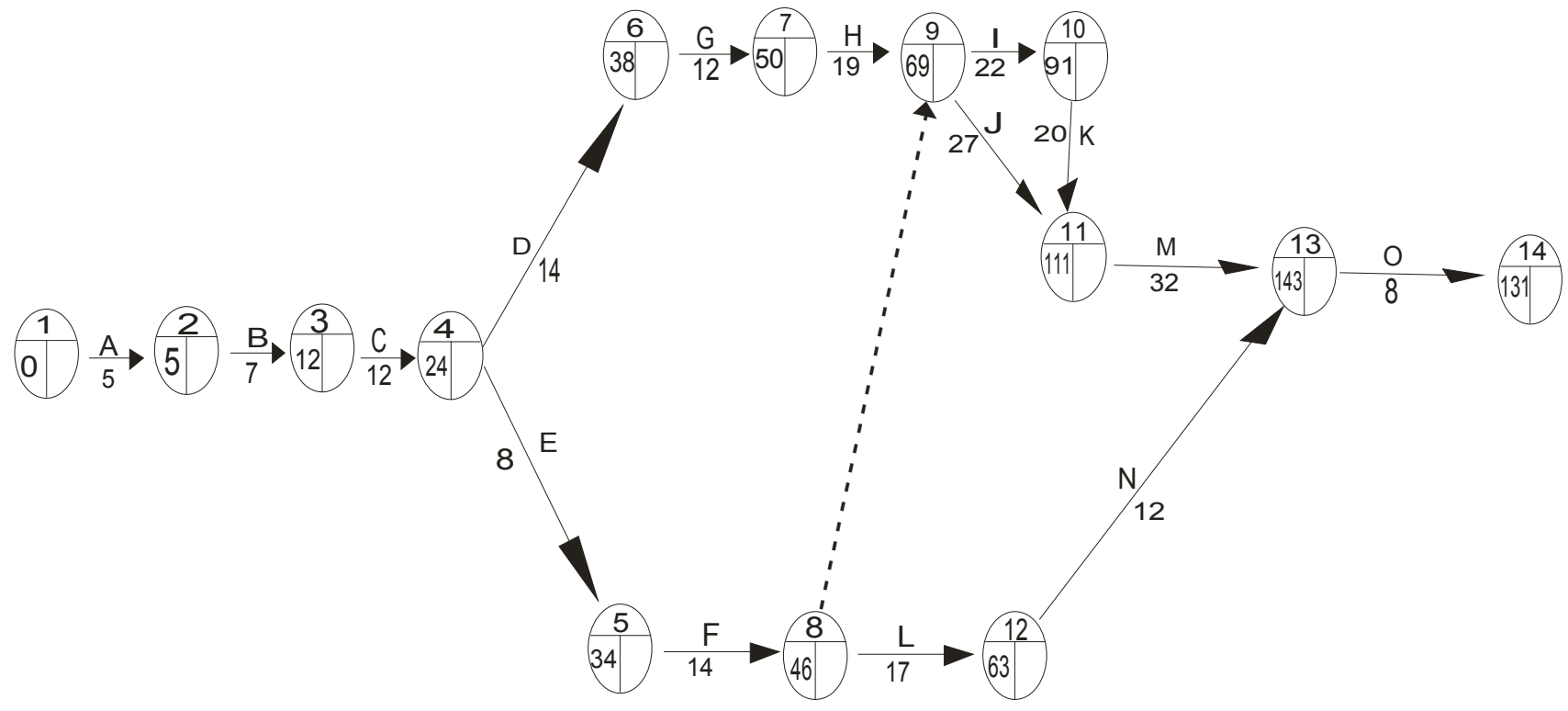

Figure 2: Forward computation network diagram with CPM.

construction project takes the total duration of 151 days as shown in row 14-column 5 of the same table. Also, activity $(8,9)$ is dummy. The network diagram of a Forward Pass computation is shown below (Figure 2):

Using equation ( 3 ), the Backward Pass computation is obtained as shown in Table 3 below

The network diagram of Backward Computation with CPM is shown below (Figure 3):

Therefore, equation (5) is used to obtain the slack values as summarized in Table 4 below. A network diagram is also drawn to indicate the sequence of activities of the critical path using CPM:

The critical path is represented by the thick lines and arcs as shown in Figure 4 below:

Network diagram is drawn in Figure 1 below to demonstrate a backward pass.

\section{Analysis of a house construction project with PERT method}

Using PERT has three times estimate from Ta- 
Table 3: Backward pass computation.

\begin{tabular}{|l|l|l|l|l|}
\hline Events & Immediate Preceeding Events & Duration & Latest Activity Time & Min. \\
\hline 14 & - & - & 151 & 151 \\
\hline 13 & 14 & 8 & $151-8$ & 143 \\
\hline 12 & 13 & 12 & $143-12$ & 131 \\
\hline 11 & 13 & 32 & $143-32$ & 111 \\
\hline 10 & 11 & 20 & $111-20$ & 91 \\
\hline 9 & 10,11 & 22 & $91-22,111-27$ & 69 \\
\hline 8 & 9,12 & 0 & $69-0,131-17$ & 69 \\
\hline 7 & 9 & 19 & $69-19$ & 50 \\
\hline 6 & 7 & 12 & $50-12$ & 38 \\
\hline 5 & 8 & 14 & $69-14$ & 55 \\
\hline 4 & 5,6 & 14 & $55-8,38-14$ & 24 \\
\hline 3 & 4 & 12 & $24-12$ & 12 \\
\hline 2 & 3 & 7 & $7-$ Dec & 5 \\
\hline 1 & 2 & 5 & $5-M a y$ & 0 \\
\hline
\end{tabular}

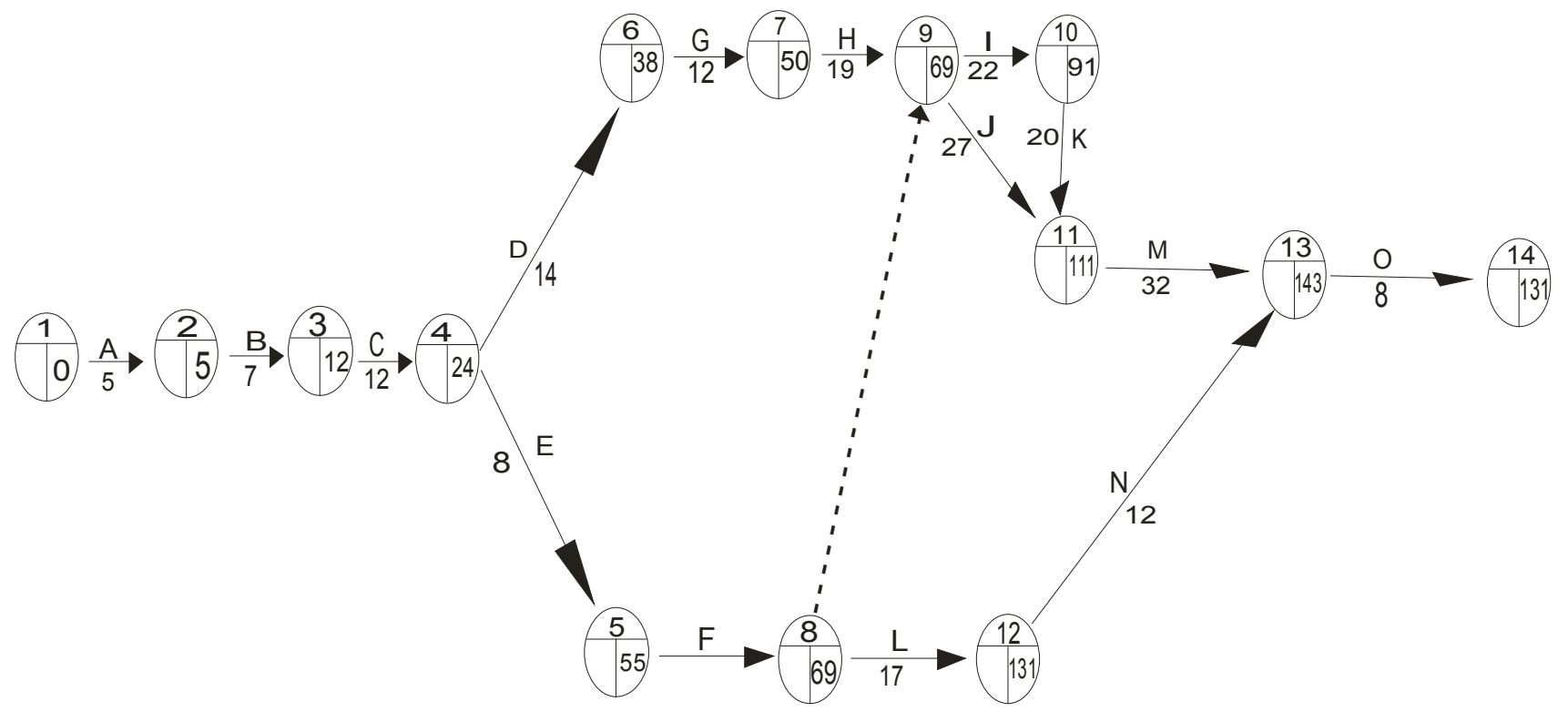

Figure 3: Backward computation network diagram with CPM.

ble 1 , the mean and variances are calculated from equation (8) and (9) respectively. And this is summarized in Table 5 below for

However, the expected project length is thus:

$\Sigma_{\text {critical }}=5+7+12+14+18+21+32+8=150$ days

The variance of the project length:

$\Sigma_{\text {critical }}=0.444+1.0+0.444+0.111+1.778+1.778+2.778+2.50+0.444+0.444=11.721$

Then $X=151$ days

Therefore, applying equation (10), the probabil- ity of the house project completion is:

prob $Z \leq\left(\frac{151-50}{\sqrt{11.721}}\right)=\operatorname{prob}[\mathrm{Z} \leq 0.30]=0.9987$

This implies that the probability that the house construction project can be completed in 151 days is $0.9987=99.87 \%$.

Hence, comparison of CPM and PERT is shown in Table 6 below:

From the table, it implies that the difference between PERT and CPM in the completion of the 


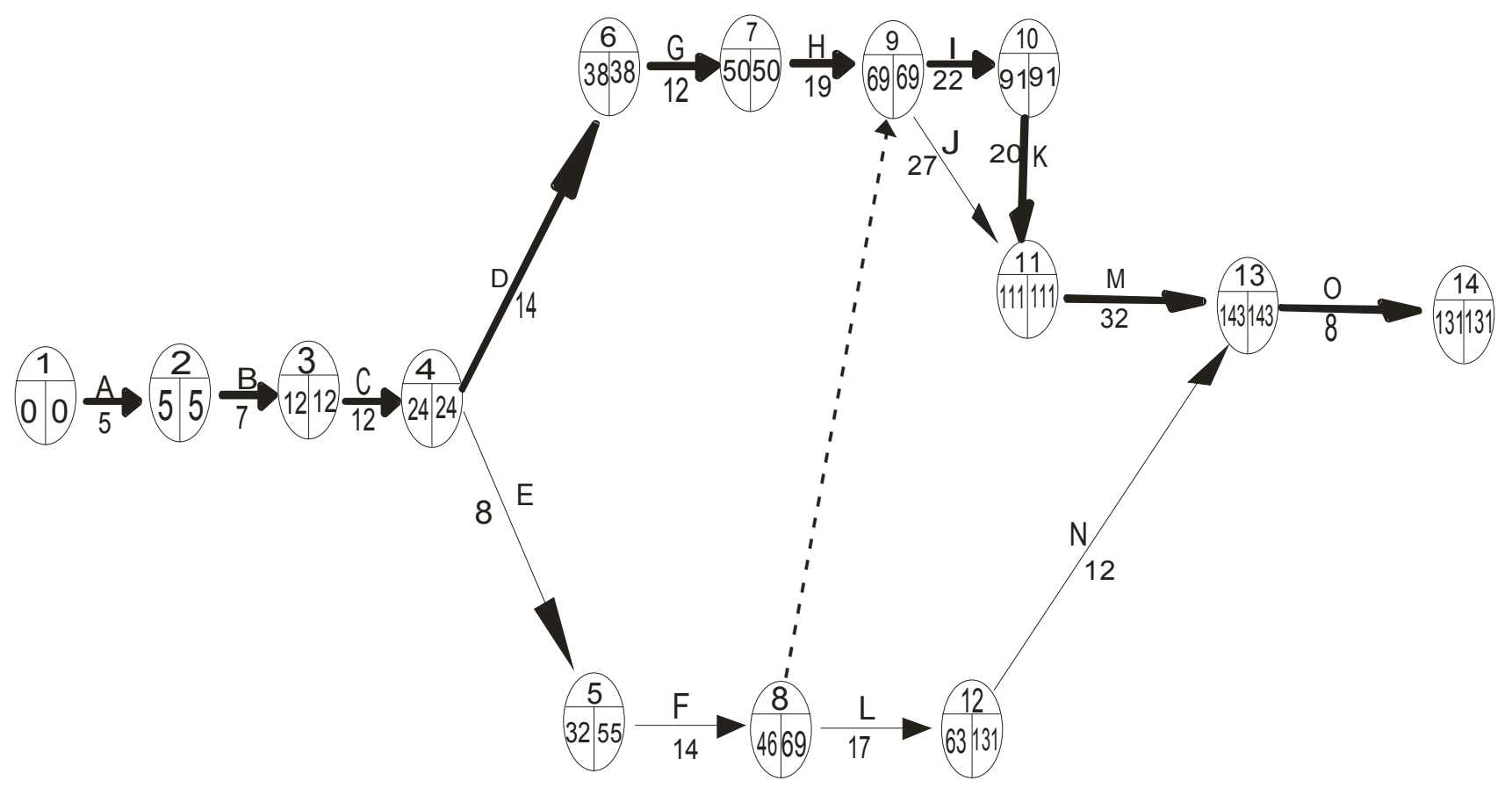

Figure 4: The critical path of the house construction project with CPM.

Table 4: The slack values.

\begin{tabular}{|l|l|l|l|l|l|l|l|l|l|l|l|l|l|l|}
\hline Events & 1 & 2 & 3 & 4 & 5 & 6 & 7 & 8 & 9 & 10 & 11 & 12 & 13 & 14 \\
\hline Latest Time & 0 & 5 & 12 & 24 & 55 & 38 & 50 & 69 & 69 & 91 & 111 & 131 & 143 & 151 \\
\hline Earliest Time & 0 & 5 & 12 & 24 & 32 & 38 & 50 & 46 & 69 & 91 & 111 & 63 & 143 & 151 \\
\hline Slack & 5 & 0 & 0 & 0 & 23 & 0 & 0 & 23 & 0 & 0 & 0 & 68 & 0 & 0 \\
\hline
\end{tabular}

Table 5: Mean and variance table.

\begin{tabular}{|l|l|l|l|l|l|l|l|}
\hline S/N & Activity & $\mathbf{i}-\mathbf{j}$ & $\mathbf{A}$ (in days) & $\mathbf{M}$ (in days) & B (in days) & Te (mean) & Var \\
\hline 1 & A & 2-Jan & 3 & 5 & 7 & 5 & 0.444 \\
\hline 2 & B & 3-Feb & 4 & 7 & 10 & 7 & 1 \\
\hline 3 & C & 4-Mar & 10 & 12 & 14 & 12 & 0.444 \\
\hline 4 & D & 6-Apr & 13 & 14 & 15 & 14 & 0.111 \\
\hline 5 & E & 5-Apr & 5 & 8 & 11 & 8 & 1 \\
\hline 6 & F & 8-May & 12 & 14 & 16 & 14 & 0.444 \\
\hline 7 & G & 7-Jun & 8 & 12 & 16 & 12 & 1.778 \\
\hline 8 & H & 9-Jul & 12 & 19 & 20 & 18 & 1.778 \\
\hline 9 & I & 10-Jul & 14 & 22 & 22 & 24 & 2.778 \\
\hline 10 & J & 11-Sep & 24 & 27 & 30 & 27 & 1 \\
\hline 11 & K & 11-Oct & 16 & 20 & 30 & 21 & 2.5 \\
\hline 12 & L & 12-Aug & 16 & 17 & 24 & 18 & 1.778 \\
\hline 13 & M & 13-Nov & 30 & 32 & 34 & 32 & 0.444 \\
\hline 14 & N & 13-Dec & 10 & 12 & 14 & 12 & 0.444 \\
\hline 15 & O & 13-14 & 6 & 8 & 10 & 8 & 0.444 \\
\hline
\end{tabular}


project is only just one day. Therefore, both CPM and PERT methods are effective and efficient in house construction.

Using equations (6) and (7) in section 3 above, Total Floats (TF) and Free Floats (FF) are obtained as shown in Table 7.

From the above Table, the sequence of activities of the critical path is A-B - C - D - G - H - I-K-M-O.

\section{Summary and Conclusion}

\section{Summary}

This work was on implementation of CPM and PERT to a house constructing project by Basse Engineering Company, Uyo, Akwa Ibom State. A descriptive study was adopted in the description of the different activities involved in the house construction project.

Secondary data were collected from a file in the management unit of Basse Engineering Constructing Company and were analyzed using CPM and PERT.

Forward and backward pass computations with

Table 6: CPM AND PERT comparison from the analyses.

\begin{tabular}{|l|l|l|l|}
\hline Comparison & Cpm & Pert & Difference \\
\hline Time (in days) & 151 & 150 & 1 \\
\hline
\end{tabular}

on CPM were obtained, and the earliest events, latest commencement and completion of activities were determined.

Both CPM and PERT methods were used in determining the slack values and the critical paths. Based on the findings, the difference existing between the implementation of CPM and PERT were seen on their three times estimates. Analysis of CPM was based on only the most likely estimate $(\mathrm{m})$, while analysis with PERT was based on optimistic estimates $(a)$, most likely estimates $(m)$ and pessimistic estimates (b).

However, PERT computation supports assigning probabilities to task completion times in accordance with the three time estimates.

Furthermore, the study showed that the total completion days using CPM was 151 days, while the total days spent using PERT was 150 days with the completion probability of a house construction project $99.87 \%$.

\section{Conclusion}

Based on the results of the analysis of this work, it can be concluded that:

PERT method is more efficient than CPM method.

The implementation of CPM and PERT in the

Table 7: TF and FF calculation in PERT.

\begin{tabular}{|l|l|l|l|l|l|l|l|l|l|l|l|l|}
\hline Activity (i, j) & Duration & & $\mathbf{t}_{\mathbf{e}}$ (mean) & Var & \multicolumn{2}{l|}{ Earliest } & Latest & FF & TF & Remark \\
\hline & $\mathbf{A}$ & $\mathbf{m}$ & $\mathbf{B}$ & & & Start & Completion & Start & Completion & & & \\
\hline $\mathrm{A}(1,2)$ & 3 & 5 & 7 & 5 & 0.44 & 0 & 5 & 0 & 5 & 0 & 0 & Critical \\
\hline $\mathrm{B}(2,3)$ & 4 & 7 & 10 & 7 & 1.0 & 5 & 12 & 5 & 12 & 0 & 0 & Critical \\
\hline $\mathrm{C}(3,4)$ & 10 & 12 & 14 & 12 & 0.44 & 12 & 24 & 12 & 24 & 0 & 0 & Critical \\
\hline $\mathrm{D}(4,6)$ & 13 & 14 & 15 & 14 & 0.11 & 24 & 38 & 24 & 38 & 0 & 0 & Critical \\
\hline $\mathrm{E}(4,5)$ & 15 & 8 & 11 & 8 & 1.0 & 24 & 32 & 47 & 55 & 0 & 23 & - \\
\hline $\mathrm{F}(5,8)$ & 12 & 14 & 15 & 14 & 0.44 & 32 & 46 & 55 & 69 & 0 & 23 & - \\
\hline $\mathrm{G}(6,7)$ & 8 & 12 & 16 & 12 & 1.78 & 38 & 50 & 38 & 50 & 0 & 0 & Critical \\
\hline $\mathrm{H}(7,9)$ & 12 & 19 & 20 & 18 & 1.78 & 50 & 69 & 50 & 69 & 0 & 0 & Critical \\
\hline $\mathrm{I}(9,10)$ & 14 & 22 & 24 & 21 & 2.79 & 69 & 91 & 69 & 91 & 0 & 0 & Critical \\
\hline $\mathrm{J}(9,11)$ & 24 & 27 & 30 & 27 & 1.0 & 69 & 91 & 84 & 111 & 15 & 15 & - \\
\hline $\mathrm{K}(10,11)$ & 16 & 20 & 30 & 21 & 2.5 & 91 & 111 & 91 & 111 & 0 & 0 & Critical \\
\hline $\mathrm{L}(8,12)$ & 16 & 17 & 24 & 18 & 1.78 & 46 & 63 & 114 & 131 & 0 & 68 & - - \\
\hline $\mathrm{M}(11,13)$ & 30 & 32 & 34 & 32 & 0.44 & 111 & 143 & 111 & 143 & 0 & 0 & Critical \\
\hline $\mathrm{N}(1,132)$ & 10 & 12 & 14 & 12 & 0.44 & 63 & 75 & 1.0 & 143 & 68 & 68 & - \\
\hline $\mathrm{O}(13,14)$ & 6 & 8 & 10 & 8 & 0.44 & 143 & 151 & 143 & 151 & 0 & 0 & Critical \\
\hline
\end{tabular}


scheduling of the house construction project is very significant on the project completion time since there is only one day difference in their completion duration.

Furthermore, the probability of completing the house project using PERT was 0.9987 which is $99.87 \%$. This implies that the possibility of completing the house project on time is very high.

\section{References}

1. Aziz RF (2014) RPERT: Repetitive project evaluation and review technique. Alexandria Engineering Journal 53: 81-93.

2. Kelly Jr JE, Walker MR (1959) Critical path planning and scheduling. Proceedings of the Eastern Joint
Computer Conference 160-173.

3. Li WG, Carter DJ (2005) Construction baseline schedule review and submitted time frame.

4. Larry Bennett (1979) A guide on critical path and critical path precedence networks.

5. Hebert JE, Deckro RF (2011) Combining contemporary and traditional project management tools to resolve project scheduling problem. Computers \& Operations Research 38: 21-32.

6. http://ir.lib.ksu.edu.tw/handle/987654321/20263

7. Moder, Joseph J (1983) Project Management with CPM, PERT and Precedence Diagramming. ( $3^{\text {rd }}$ edn), Van Nostrand Reinhold, New York. 